\title{
Faoladh : A Case Study in Cinematic VR Storytelling and Production
}

\author{
Declan Dowling ${ }^{1}$, Colm O Fearghail ${ }^{2}$, Aljosa Smolic ${ }^{2}$, and Sebastian Knorr ${ }^{2,3}$ \\ 1 Tile Films Ltd, Ireland \\ declan.t.dowling@gmail.com \\ 2 V-SENSE, School of Computer Science and Statistics, Trinity College Dublin, The \\ University of Dublin, Ireland \\ \{ofearghc, smolica\}@scss.tcd.ie \\ 3 Communication Systems Group, Technical University of Berlin, Germany \\ knorr@nue.tu-berlin.de
}

\begin{abstract}
Portraying traditional cinematic narratives in virtual reality (VR) is an emerging practice where often the methods normally associated with cinematic storytelling need to be adapted to the $360^{\circ}$ format. In this paper we investigates some proposed cinematic practices for narrative storytelling in a cinematic VR film set in late 9th century Ireland that follows the perilous journey young Celt as he evades being captured by Viking raiders. From this we will analyze the fidelity of those practices with results collected from YouTube Analytics.
\end{abstract}

Keywords: 360-video; virtual reality; storytelling; cinematography; YouTube Analytics

\section{Introduction}

In contrast to traditional cinema, where the viewer perceives the world through a window i.e. the cinema screen, cinematic VR allows a person to be present within the world by wearing a head-mounted display (HMD) [2]. This poses a new challenge for filmmakers as it necessitates the expansion of cinematic language to an immersive, border-less format. In this paper, we introduce a case study in cinematic VR storytelling and production for the VR film Faoladh [1]. We investigate some proposed cinematic practices for narrative storytelling in a cinematic VR film set in late 9th century Ireland that follows the perilous journey young Celt as he evades being captured by Viking raiders.

\section{Adapting Cinematic Storytelling to VR}

Four techniques that have traditionally formed the 'tools' that filmmakers rely on to tell their stories are: cinematography, sound, editing and mise-en-scene [3]. The expansion of these tools into VR, however, requires each to be re-evaluated. 
Modern cinematography often uses as combination of light, movement, angles and focus to tell a story. With VR the viewer takes control of the camera and can look in any direction so angles become less effective narrative devices while camera position and movement become more critical to the viewers perception and level of comfort. A popular method for focusing viewer attention in traditional cinema is depth of field (DOF) which can draw attention to aspects of the scene in relation to its distance from the camera. VR cameras like the Google Odyssey used in this production, often rely on small sensor arrays with little to no DOF. Although it is possible to simulate DOF in post production via stereoscopic disparity, without the frame of the cinema screen it is a much less effective tool for focusing viewer attention in VR.

Spatial audio can be an effective tool to guide the viewer to another area of the scene as are directional cues by the actors present within, but VR also poses technical challenges in terms of staging and performance due to restrictions in editing. Quick cuts can disorient the viewer necessitating longer takes which results in staging and performances more closely aligned to traditional theater and deep focus cinematography which became quite popular in the 1940s with films such as Orson Welles Citizen Kane.

\section{Deep focus staging}

In Figures 1 and 2 we see a staging plan and the resulting shot from a pivotal scene where the hero stares directly towards the dramatic death of the monk stage right, while a line is drawn from the villains arrow towards the hero leaving only a single prop the user can interact with to block its path. The objective is to draw the viewers attention from the hero $>$ drama $>$ threat $>$ resolution.

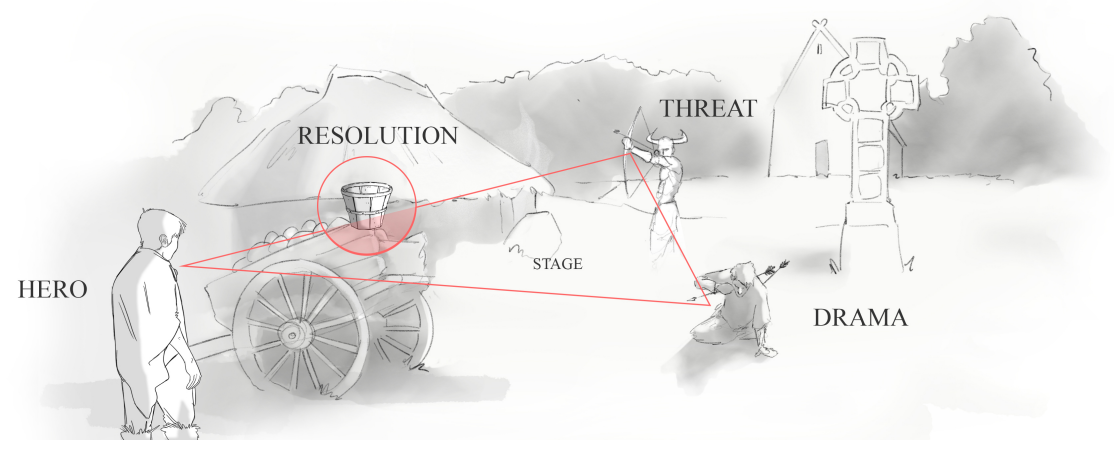

Fig. 1. Staging plan

In Figure 2 a heatmap collected from over 200 viewers is additionally overlaid to the shot, which shows a focus on the threat and solution with little attention is given to the hero or drama. From this we can conclude that the position of the camera, its proximity to the hero and the fact we can't see his face creates 


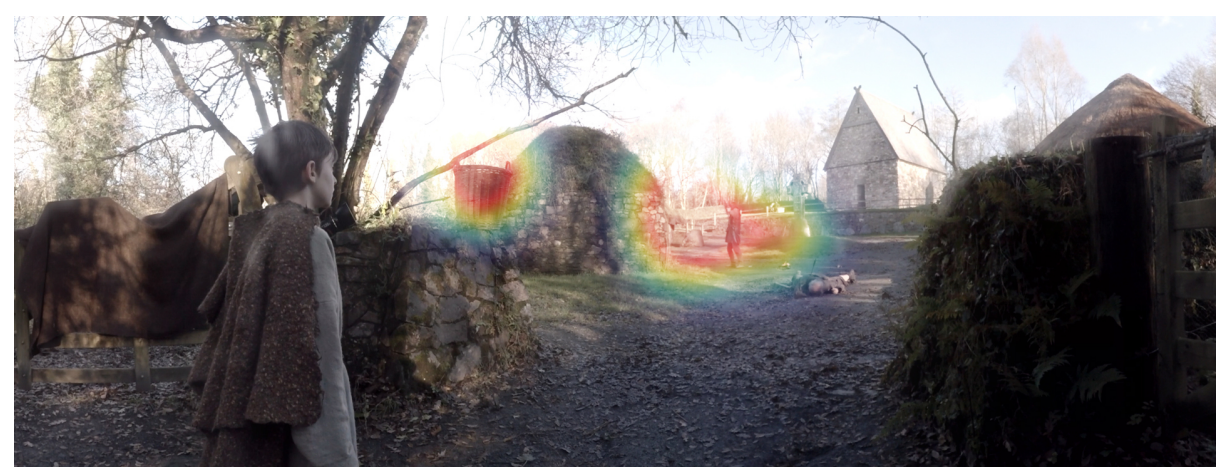

Fig. 2. A YouTube Analytics heatmap collected from over 200 viewers

a narrative cue similar to a traditional over shoulder or 3rd person perspective where the viewer will look in the direction the hero is facing as opposed to at the hero.

\section{Narrative Pacing}

Filmed at the Irish National Heritage Park, a three-act story was built around each of the parks main locations. To reduce cuts and mitigate audience fatigue, a continuous path was plotted with intermediate rest stops throughout (see shoot plan in Figure 3).

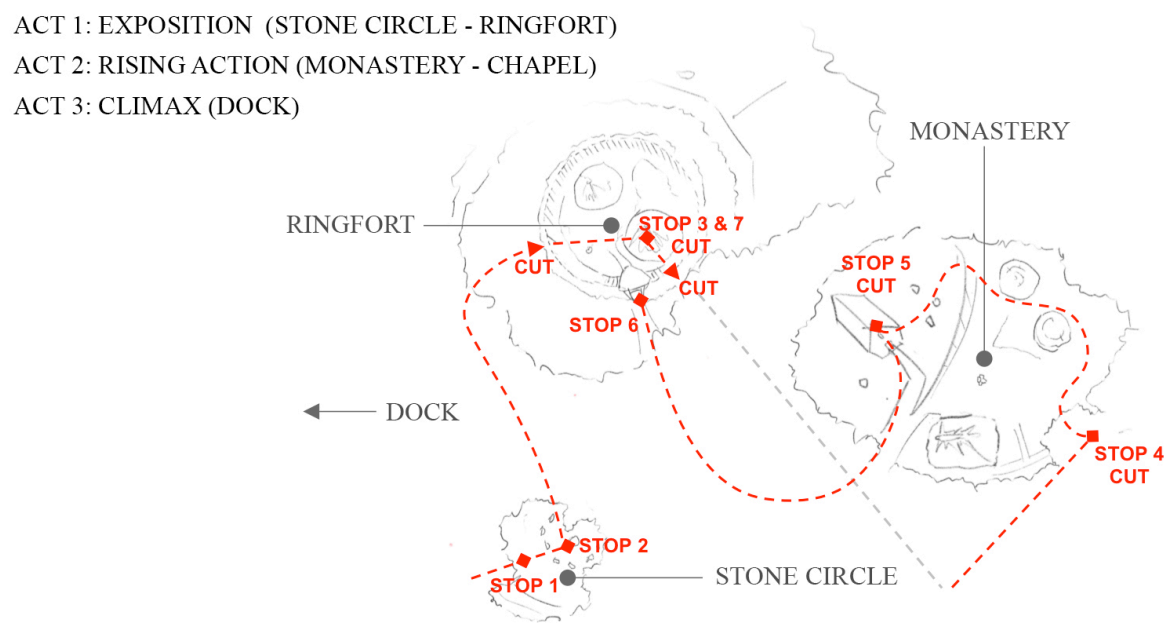

Fig. 3. Shoot plan at the Irish National Heritage Park 
A YouTube Analytics chart of relative audience retention (see Figure 4) shows audience interest peaks at 3:27 which corresponds with the scene from section 3 but is shortly followed by a dip in retention which coincides with a lull in the rising action portion of the film at 3:55 (see Stop 5 in Figure 3).

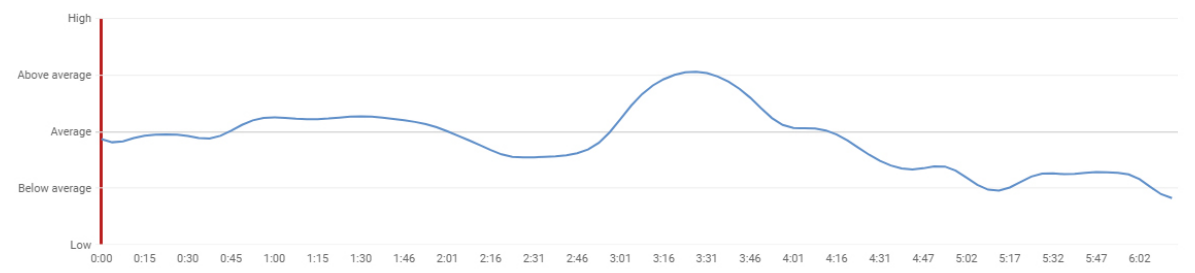

Fig. 4. YouTube Analytics chart of relative audience retention

Relative audience retention shows a YouTube video's ability to retain viewers during playback by comparing it to all YouTube videos of similar length. When the graph is higher, it indicates how many more viewers kept watching the video for that timeframe compared to the same timeframe in other YouTube videos ${ }^{4}$.

\section{Conclusion}

Giving the audience the ability to look in any direction and accompanying this with a perceived sense of presence can cause underlying narrative cues such as the 3rd person effect or a lull in pacing to disrupt narrative flow and interrupt storytelling in cinematic VR. Further investigation is required to list all possible adaptations of cinematic practices and the underlying narrative devices virtual reality can reveal.

\section{Acknowledgment}

This publication has emanated from research supported in part by a research grant from Science Foundation Ireland (SFI) under the Grant Number 15/RP/2776.

\section{References}

1. Faoladh - http://faoladh.net/

2. Smith, S., Marsh, T., Duke, D., Wright, P.: Drowning in immersion. Proceedings of UK-VRSIG 98, 1-9 (1998)

3. Vosmeer, M., Schouten, B.: Project Orpheus a research study into $360^{\circ}$ cinematic VR. In: Proceedings of the 2017 ACM International Conference on Interactive Experiences for TV and Online Video. pp. 85-90. TVX '17, ACM, New York, NY, USA (2017), http://doi.acm.org/10.1145/3077548.3077559

\footnotetext{
${ }^{4}$ https://support.google.com/youtube/answer/1715160
} 